\title{
Responsabilidades adultas en la educación y el cuidado infantil Discusiones histórico-etnográficas
}

\section{(1) Laura Cerletti*y Laura Santillán**}

Recibido octubre de 2017

Aceptado marzo de 2018

Resumen

En el campo de la educación e intervención acerca de la infancia, en la actualidad, se ha hegemonizado un discurso que instala un reparto de responsabilidades adultas relativamente novedoso y que exalta la figura parental como condición del bienestar y buen desarrollo de la niñez. Acorde con una perspectiva antropológica, observaremos el carácter historizado de esta construcción, y las significaciones y disputas que tienen lugar en el campo de la vida práctica. En este trabajo nos proponemos analizar algunas continuidades y rupturas en las representaciones hegemónicas sobre las responsabilidades adultas en torno a la educación y crianza de los niños, así como las diversas apropiaciones que se producen en los escenarios cotidianos de actuación. Nos basaremos principalmente en los registros etnográficos realizados en nuestros procesos de investigación, para los cuales se llevó a cabo trabajo de campo en la ciudad de Buenos Aires y en el área aledaña denominada Gran Buenos Aires.

\section{Adult responsibilities in children's education and care. Historical- ethnographic discussions}

\footnotetext{
Abstract

In the field of education and intervention on childhood, contemporarily, a discourse establishing a relatively new distribution of adult responsibilities that enhances parental figures as a condition for children's wellbeing and development has become hegemonic. According to an anthropological perspective, in this paper we analyze continuities and change in hegemonic representations of adult responsibilities regarding the education and upbringing of children, as well as the diverse appropriations that take place in everyday-life contexts. Analysis is based on the ethnographic accounts constructed

* Doctora en Antropología (UBA), investigadora CONICET (categoría Adjunta) y docente de la Facultad de Filosofía y Letras. Programa de Antropología y Educación (Sección de Antropología Social, Instituto de Ciencias Antropológicas, Facultad de Filosofía y Letras, UBA). Buenos Aires, Argentina. Correo electrónico: laurabcerletti@yahoo.com.ar ** Doctora en Antropología (UBA), investigadora CONICET (categoría Independiente) y docente de la Facultad de Filosofía y Letras. Programa de Antropología y Educación (Sección de Antropología Social, Instituto de Ciencias Antropológicas, Facultad de Filosofía y Letras, UBA). Buenos Aires, Argentina. Correo electrónico: laursantillan@ gmail.com
}

\section{Key words}

Parental Responsibility; Children's Education and Care; Hegemonic Regulations; Appropriations; Historical-Ethnographic Approach
Palabras clave

Responsabilidad parental; Educación y cuidado infantil; Regulaciones hegemónicas; Apropiaciones;

Enfoque histórico-etnográfico 
throughout our research processes, during which fieldwork was conducted in the City of Buenos Aires and its suburbs.

\section{As responsabilidades adultas em educação e a assistência à infân- cia. Discussões histórico-etnográficas}

\section{Resumo}

Palavras-chave

Responsabilidade parental; Educação e assistência à infância;

Regulamentos hegemônicos; apropriacões; Abordagem históricoetnográfica
1. En nuestros planteos, el término crianza, a diferencia de la tendencia presente en estudios sociológicos... (continúa en página 100)

2. En adelante, utilizaremos genéricamente la palabra "niños" para aludir también a las niñas.

3. La categoría "frente discursivo" alude a la articulación de discursos que -en torno a un mismo tema- surgen de la negociación entre diversos grupos de interés y que en buena medida tienden a reificar una serie de preocupaciones y presupuestos asociados a dicho frente discursivo (Fonseca y Cardarello, 2006). En otros trabajos hemos abordado diversos aspectos de la conformación de este frente discursivo (Cerletti y Santillán, 2013; Cerletti, 2015; Cerletti y Gessaghi, 2017).
Na atualidade, no domínio da educação e da intervenção em crianças, tem sido hegemonizado um discurso que instala um reparto de responsabilidades relativamente novo, que exalta a figura parental como uma condição de bem-estar e o desenvolvimento das crianças. Em concordância com uma perspectiva antropológica, vamos pensar o caráter histórico de sua construção, bem como os significados e disputas que ocorrem no domínio da vida prática. Assim, neste artigo, analisaremos algumas das continuidades e rupturas nas representações hegemônicas sobre as responsabilidades dos adultos na educação e na criação dos filhos, assim como as apropriações que ocorrem nas situações corriqueiras. Vamos basear-nos sobre os registros etnográficos realizados em nossos processos de pesquisa; o trabalho de campo foi realizado na Cidade de Buenos Aires e na áreas de Grande Buenos Aires.

\section{Introducción}

Abrir interrogantes acerca de los procesos de producción de las responsabilidades y obligaciones adultas en torno a la educación y la crianza ${ }^{1}$ implica asumir como punto de partida que no hay ninguna base de sustentación - hecho demostrado ampliamente desde la antropología y otras ciencias sociales- para suponer que la filiación directa determina el cuidado sobre los niños. Sólo se trata de modalidades sociohistóricamente configuradas en las que una sociedad - la nuestra, en este casoconcreta aspectos clave de la formación y reproducción (en sentido amplio) de las nuevas generaciones, que distan de ser naturales o inmutables.

A partir de nuestras investigaciones histórico-etnográficas sobre los procesos de educación de los niños y niñas, ${ }^{2}$ hemos documentado con suma recurrencia, dentro y fuera de los espacios escolares, que se suele aludir de maneras específicas y reiteradas a la responsabilidad parental que ubican en medio de la escena a los progenitores de los niños - con centralidad en las madres-y que situarían a dicha responsabilidad como condición exclusiva para el pleno desarrollo y bienestar de los niños. De manera progresiva, y en función de la socialización de los niños, sus familias y contextos de origen empezaron a ser considerados como requisito prioritario para el logro de una exitosa escolaridad (Neufeld y Thisted, 2004; Achilli, 2010; Nogueira, 2011; Carmona, 2014); y simultáneamente, ya en términos más amplios, son representados como garantía sine qua non del buen desarrollo, la salud y bienestar de los niños, tal como es posible de documentar en líneas programáticas e intervenciones vinculadas con la primera infancia (Santillán, 2016).

En efecto, lo que constatamos es que en las últimas décadas, en el campo educativo y de intervención sobre la infancia - en paralelo con otros campos de la vida social- se ha consolidado un frente discursivo (Fonseca y Cardarello, 2006), ${ }^{3}$ en cuya producción 
tienen protagonismo determinadas agencias nacionales - ministeriales y no- $-\mathrm{y}$ organismos internacionales, que hegemoniza las formas enunciadas como "correctas" de ser familia, y que se centra especialmente en las figuras parentales.

Si bien las referencias a los contextos familiares de los niños - sus condiciones y potencialidades para el cuidado - tempranamente han formado parte del campo de intervenciones de la infancia en nuestro país y en nuestra región (Moreno, 2002; Carli, 2005), nos referimos a regulaciones que se destacan por algunos rasgos específicos. Una singularidad es que, en la actualidad, las modelaciones de los comportamientos adultos se anudan a renovadas interpelaciones a la presencia (física) y participación de las familias, y a la generación de particulares expectativas en cuanto a los modos en que los grupos domésticos deben interactuar con las escuelas y con otros espacios sociales vinculados con los niños. A la vez, estas regulaciones incluyen un conjunto de sugerencias respecto de las acciones a realizar en el ámbito doméstico, entre las cuales se destacan la demostración de interés y el acompañamiento. Sin embargo, hemos documentado que estas formas-así como son frecuentemente enunciadas por docentes y distintos sujetos vinculados de lleno a la educación infantil— son también activamente apropiadas, a la vez que discutidas, tensionadas y resignificadas por los mismos padres y/u otros adultos con niños a su cargo en el ámbito doméstico, e incluso por los mismos docentes, lo cual no implica en sí ninguna dificultad o contrariedad para los niños.

Diversos estudios han contribuido a comprender el carácter construido y contextualizado de la atribución de responsabilidades en torno a los niños. Nos referimos tanto a trabajos que, desde un enfoque sociojurídico, han discutido y analizado las nociones contemporáneas de responsabilidades parentales (Harris, 2009; Di Nella, 2016), como a algunos que han discutido los modos de configuración de las responsabilidades adultas desde un enfoque antropológico y sociológico (Díaz de Rada, 2003; Ochs e Izquierdo, 2009; Ochs y Kremer-Sadlik, 2013), atendiendo a la perspectiva de los mismos niños (Edwards y Alldred, 2000). Sin embargo, constituye una vacancia comprender el carácter historizado de esta construcción, así como las significaciones y disputas que tienen lugar en el campo de la vida práctica. De tal forma, en este trabajo avanzaremos en el reconocimiento de algunas continuidades y rupturas en las representaciones hegemónicas sobre las responsabilidades adultas en torno a la educación y crianza de los niños, y ahondaremos en los sentidos que las transformaciones asumen, como así también en las huellas de su genealogía. Para eso, con base en los aportes que ofrece el abordaje de corta y mediana duración (Neufeld, 1997; Rockwell, 2009), nos centraremos en algunos hitos que se produjeron a partir de mediados del siglo XX y que son relevantes en los procesos de construcción contemporáneos. Investigaciones en el ámbito local han documentado distintos aspectos vinculados con la historia de la relación familias-escuelas (Cragnolino, 2000; Neufeld, 2000; Neufeld y Thisted, 2004; Santillán, 2009; Achilli, 2010; Cerletti y Santillán, 2013; Cerletti, 2015) y sentaron un antecedente importante para el tema. En consonancia con las investigaciones mencionadas, nuestra indagación contempla como cuestión nodal un abordaje del presente en términos historizados (Sanjek, 1991), y articula los conocimientos sobre los principales procesos sociohistóricos acaecidos durante las últimas décadas con lo registrado en el trabajo de campo (Achilli, 2005). Asimismo, procuraremos dar cuenta de la variedad y complejidad con que se desenvuelven la crianza y educación, particularmente en el campo de atribución de responsabilidades y de las prácticas cotidianas.

En función de este análisis, recuperamos los registros de las observaciones y entrevistas que venimos realizando en nuestros procesos de investigación, para los cuales se llevó a cabo trabajo de campo en la Ciudad y el Gran Buenos Aires. Nos referimos, por un lado, a la investigación que, situada en la ciudad de Buenos Aires primero, y luego en el conurbano bonaerense norte ${ }^{4}$, viene focalizando desde el año 2004 en las relaciones entre las familias y las escuelas, con atención a las representaciones y prácticas 
5. Dicha investigación está a cargo de Laura Santillán.

6. Nos referimos a los proyectos de sucesivas programaciones UBACyT (2010-2012; 2010-2014; 2014-2017, FFyL, UBA), PIP (CONICET) y PICT/FONCYT (Ministerio de Ciencia y Tecnología) en curso actualmente, radicados en el Programa de Antropología y Educación de la Sección de Antropología Social (del Instituto de Ciencias Antropológicas, Facultad de Filosofía y Letras, Universidad de Buenos Aires). Componen estos equipos de investigación los siguientes investigadores, tesistas de grado y posgrado: Agustín Barna, Marcela Bilinkis, Julieta Calderón, María Laura Fabrizio, Soledad Gallardo, Juliana Montero y Florencia Soto. 7. En los proyectos colectivos de investigación en curso, la indagación de estas cuestiones contempla dimensiones diversificadas de la experiencia de los niños (escolarización inicial y primaria, educación sexual, protección de derechos, formación musical, educación y cuidado), así como las prácticas y sentidos desplegados por los adultos vinculados a ellos/as, en escenarios distintivos de actuación (estatales, familiares, comunitarios, consultorio psicopedagógico),

los cuales documentamos a partir de un trabajo de campo sostenido en el tiempo en el sur de la ciudad de Buenos Aires y en distritos de la zona oeste (Matanza), sur (Almirante Brown), norte y noroeste del Gran Buenos Aires (Vicente López, Escobar y Tigre).

8. El uso de comillas acá y en las frases siguientes señala el uso de categorías sociales o nativas (Rockwell, 2009), registradas con recurrencia en nuestro trabajo de campo. de los docentes y los adultos con niños a su cargo en el ámbito doméstico (pertenecientes tanto a los sectores subalternos como a la clase media), que ha profundizado asimismo en una línea más específica de historización de los discursos vigentes sobre el tema. Por otro lado, aludimos a la investigación que se viene realizando desde el año 2001 en el Gran Buenos Aires, ${ }^{5}$ con foco en las dimensiones políticas y sociales que se ponen en juego en espacios colectivos (estatales y de la sociedad civil) —entre las cuales se destaca una serie de iniciativas levantadas por los pobladores de los barriosque tienen como objetivo la educación y el cuidado de la infancia. Asimismo, el eje de interés del artículo se inscribe y da continuidad a las preocupaciones que venimos abordando en los proyectos colectivos de investigación en los cuales participamos como directoras. ${ }^{6}$ En ellos, hemos dado centralidad a los sentidos y modalidades que se construyen y dirimen en torno a las responsabilidades adultas a nivel societal y discursos más generales, como así también en los escenarios cotidianos de interacción, ${ }^{7}$ cuestiones que en conjunto nos conducen a desnaturalizar la universalidad y necesariedad de los requerimientos epocales referidos.

Como expusieron otros autores, bajo este propósito de historizar nuestro "presente etnográfico" está el interés de hacer visibles y legibles los sentidos que ha dejado el curso de la historia en aquello que investigamos (Batallán, 2007; Rockwell, 2009), lo cual permite problematizar el carácter natural de las categorías y atributos tal como se presentan en la "realidad" (Neufeld, 1997).

Por tanto, en el siguiente apartado reconstruiremos los hitos a partir de los cuales entendemos que se ha ido consolidando, a lo largo de varias décadas, el frente discursivo aludido. Luego, avanzaremos sobre las diversas formas en que la educación y el cuidado infantil se despliegan cotidianamente en los contextos familiares, según hemos registrado a lo largo de nuestros trabajos de campo. Finalmente, abordaremos distintos modos en que los sujetos directamente vinculados a los niños en sus ámbitos de vida próximos producen sentidos en torno a sus propias responsabilidades respecto de la educación y el cuidado infantil, que evidencian tanto apropiaciones como producciones de sentidos que confrontan con aquellos hegemonizados en la actualidad.

\section{Responsabilidades parentales en torno a la educación y el cuidado infantil: sobre la conformación de un frente discursivo}

¿Qué sentidos específicos asumen las responsabilidades parentales en la actualidad? La apelación a las responsabilidades adultas constituye un tema que preocupa cada vez más y que diversas agencias, gubernamentales o no gubernamentales, difunden por diversos medios, tanto dentro como fuera de la escuela: carteleras, cartillas, campañas, informes y publicaciones de importante alcance. Asiduamente, desde distintos organismos, se enuncian una serie amplia de acciones acerca de lo que deberían hacer los padres de los niños como requisito para su pleno desarrollo y formación. En relación con la escolaridad, estas referencias vuelven de diverso modo a un conjunto de requerimientos que se presentan como insoslayables: la necesidad de "sentarse" 8 con los niños en el ámbito doméstico para hacer tareas o supervisar el uso de cuadernos y carpetas utilizados por ellos en la escuela, la realización en los hogares de determinadas actividades específicas que acompañen los contenidos trabajados en las aulas, también juegos (con números, canciones, dibujos, etc.), la provisión de diversos útiles y materiales, así como la búsqueda de información complementaria. En términos más amplios — quedando aquí incluidos los niños de más corta edad—, las recomendaciones se centran en la necesidad del cumplimiento de los horarios de entrada y salida previstos por las instituciones, la asistencia a las "reuniones de padres" y a otras citaciones (sean periódicas o extraordinarias); en que los niños - cuando están fuera de la escuela - sean cuidados por adultos (preferentemente la madre) y no por otros niños, 
que dentro de la vida familiar se cumplan determinadas pautas de horarios (de sueño, de recreación, etc.) y de alimentación (también con horarios delimitados, privilegiando la comensalidad compartida, entre otras cuestiones).

Aunque gestados desde diversas fuentes, tomados en conjunto en relación con este frente discursivo, esos lineamientos sobre las "responsabilidades adultas" en buena medida se resumen en las categorías de "participación” y "acompañamiento" (Cerletti, 2014; Santillán, 2016; Cerletti y Gessaghi, 2017). Sin embargo, según hemos evidenciado a lo largo de nuestras indagaciones, ni la realización de esas acciones garantiza una educación o escolarización más efectiva, ni lo contrario (Santillán, 2012; Cerletti, 2014). Es decir, no se puede sostener una relación de causalidad directa entre las acciones adultas y los resultados en los niños. Como todo fenómeno social, las premisas - vueltas "verdades" - que establece este frente discursivo no son naturales, ni obvias, ni autoevidentes. A fin de avanzar en la desnaturalización de estos lineamientos, entonces, encontramos necesario atender a la historicidad de estos planteos, que debemos situar en vinculación con procesos inscriptos en contextos sociales determinados.

Cuando atendemos al campo escolar, este acompañamiento y presencia física de hecho no sucedía hasta mediados del siglo XX (Amuchástegui, 2000). Tampoco se encuentran alusiones en las publicaciones especializadas (anteriores a las últimas décadas) a este requerimiento contemporáneo (Santillán, 2009; Cerletti, 2015). Asimismo, al indagar con mayor profundidad en las trayectorias de sujetos pertenecientes a diversas generaciones, hemos podido documentar que estas exigencias no han existido desde siempre. Qué les corresponde hacer a los docentes, qué a los padres, qué a otros sujetos vinculados a los niños, ha implicado debates, discusiones y transformaciones históricas, como venimos planteando. De tal forma, al indagar sobre el carácter construido de este cambio, se evidencia la productividad social que han tenido los discursos de los organismos internacionales.

En nuestro país, a partir de la década de 1980 - en consonancia con el retorno de la democracia-, los lineamientos de estos organismos comenzaron a cobrar peso en orientaciones que estamos marcando como vinculadas al campo de la educación y la infancia. Desde esa década, pero con mayor énfasis en los años noventa, los organismos internacionales vienen haciendo explícita - en ocasiones hegemonizando el campo educativo en detrimento de otras propuestas - la necesidad de concretar el desarrollo integral de los niños y jóvenes a través de acciones que hagan fuerte hincapié en la promoción y la participación social. ${ }^{9}$ La "participación" — referenciada con el fin del gobierno de facto en la democratización en distintas esferas sociales, aunque luego coadyuvando y dando legitimidad a la transferencia de responsabilidades a los planos individuales de acción en los noventa- se configura, junto con la de acompañamiento e interés, como una de las categorías clave con la que estos organismos indican a los diversos adultos involucrados en la educación infantil formas de actuar e interactuar entre sí (Cerletti, 2014). En efecto, publicaciones tales como las producidas por UNICEF (2002, 2009, entre otros) y UNESCO (2004, 2012, entre otros) se tornan paradigmáticas en relación con el planteo de que la "participación” y el "acompañamiento" parental mejoran los resultados escolares y el desarrollo de los niños (Cerletti y Santillán, 2013; Santillán, 2014; Cerletti y Gessaghi, 2017). Asimismo, según lo hemos documentado también, en línea de continuidad con esto, en recomendaciones presentes para la atención a la primera infancia y en relación con "el buen comienzo" (UNICEF, 2005; OIT, 2012), puntualizaciones relativas a la "socialización primaria" vuelven a colocar al núcleo familiar en el eje central de la escena de los cuidados y el adecuado desarrollo de los niños, en tanto "entorno seguro" (Santillán, 2016). Aludimos a nociones sobre la familia que, según documentamos, reifican la visión de la necesidad de que estas demuestren "recortes claros" y roles claramente definidos (Cerletti, 2014; Santillán, 2016).
9. Vale decir que, por parte de los organismos multilaterales, las recomendaciones en relación con la participación comunitaria no son nuevas. Para nuestra región de América Latina, al menos desde la década del sesenta, organismos internacionales como la CEPAL y la ONU se han encargado de presentar a la participación social como una actividad "necesaria" para lograr la resolución de problemas de integración en áreas específicas -y estratégicas-como son la salud, la educación y la economía (Menéndez y Spinelli, 2006). 
10. Eva Giberti es psicóloga y asistente social, $y$ ha tenido una larga y profusa producción en distintas áreas. Entre 1961 y 1973 publicaba periódicamente una columna en el diario La Razón, que luego compiló en cuatro tomos, bajo el título de Escuela para padres. Además de la extensa difusión de esta obra-que, según Plotkin (2003), vendió más de 150.000 ejemplares-, condujo programas de radio y televisión, entre muchas otras actividades, con lo cual se ha configurado como una de las referentes de la divulgación de las teorías "psi" en nuestro país.

11. Nuevamente, sólo a modo de punteo, nos interesa mencionar que en el mismo período aludido acá se produjeron dos procesos sumamente significativos desde el punto de vista de la definición de los lineamientos de las políticas educativas (con su contracara respecto de la definición de responsabilidades y obligaciones en torno a la infancia). Nos referimos tanto a los inicios de la descentralización del sistema educativo (que comenzó a transferir la responsabilidad educativa a planos jurisdiccionales locales), y a los avances en la injerencia de la Iglesia católica en materia educativa (véase Cerletti, 2012).
Sin embargo, si bien la apelación a la "participación" condensa en buena medida todo un conjunto de formas de regular la acción parental y la distribución de obligaciones adultas (entre padres y educadores), los supuestos en torno al cuidado de los niños y las condiciones necesarias para su desarrollo (con sus implicancias en la escolarización, entre otras cuestiones), exceden a esta apelación. De hecho, al preguntarnos con mayor profundidad respecto de los supuestos y sobreentendidos sobre los que se sustenta el frente discursivo hegemónico aludido, registramos que era necesario ampliar la indagación a períodos históricos anteriores. Y con ello, nos introducimos en otro hito histórico: la década de 1960 y la amplitud con que se divulgaron teorías, como las "psi", en ese momento (Cerletti, 2015), tendientes a construir una determinada "disponibilidad" afectiva de los adultos más cercanos a la vida de los niños.

Hasta la década del sesenta, y en relación con uno de los aspectos de la educación como es la escolaridad, hemos documentado cómo la exigencia hacia los padres giraba fundamentalmente en torno al cumplimiento de la obligatoriedad, como ya mencionamos (Santillán, 2009). Si un niño era hijo de padres "sanos" (en términos físicos y morales), nada debería impedir que la escuela pudiera cumplir con su trabajo. Sin embargo, como venimos planteando, actualmente se ha instalado con toda fuerza la idea de que si la familia no "socializa" correctamente, la escuela no puede hacer su parte. Por cierto, con la difusión masiva de las teorías "psi" se ampliaron a un conjunto cada vez mayor de la población ciertos supuestos que reubicaron la responsabilidad parental en la salud psíquica infantil (Carli, 1997; Cosse, 2010; Cerletti, 2015). Las acciones adultas ya no deberían garantizar solamente la moral, la salud física del niño y su buena constitución, sino también ocuparse - con énfasis en la madre - de la "higiene mental" (como la denominaba Eva Giberti) ${ }^{10}$ de los niños, que sería determinante del pleno desarrollo del "hombre adulto". Así, si bien no hay mucho de novedoso en el hecho de que hubiera toda una serie de saberes, discursos y dispositivos dirigidos a que los padres (y más aún, las madres) cumplieran con sus responsabilidades y obligaciones respecto de los niños (Nari, 2004; Darré, 2013), sí se instaló una nueva concepción relativa a cuáles deberán ser esas responsabilidades. La importancia del amor materno y los límites, la evitación de que los conflictos adultos lleguen a los niños, la condena a los castigos físicos, el reparto de roles al interior de la pareja en términos de "funciones" psicológicas (no ya restringidas a la provisión y el cuidado dado por hombres y mujeres respectivamente) son algunos de los tópicos que se empezaron a introducir con la divulgación de estas teorías (Plotkin, 2003; Cosse, 2010; Rustoyburu, 2010; Cerletti, 2015). Un niño bien cuidado ya no sería simplemente un niño limpio y alimentado, sino que sería uno cuya particularidad psicológica debería ser conocida (y aprendida, según lo transmitieran los expertos del campo "psi") y bien manejada por sus padres (Cerletti, 2015).

En el marco de lo que muchos autores caracterizaron como la "psicologización" de la infancia, se produjo un giro relevante en cuanto a las explicaciones del desarrollo del niño y en relación con el "éxito" - o fracaso- escolar que se centró en nuevas directrices, tales como las vinculaciones íntimas y la armonía familiar (Carli, 1997). Si bien por razones de espacio no podemos profundizar en los motivos que dieron lugar a este proceso de "psicologización" de la infancia ni a otros procesos contemporáneos que también han dejado profundas huellas en la temática que trabajamos, ${ }^{11}$ sí nos interesa señalar cómo fue teniendo lugar, de esta manera, una reconfiguración de los modos en que se representan las obligaciones y responsabilidades adultas en torno a los niños.

En resumen, nos interesa apuntar a estos dos procesos que identificamos por su relevancia para entender la constitución contemporánea del frente discursivo aludido. Nos referimos, por un lado, al proceso de difusión de las teorías "psi” a mediados del siglo $\mathrm{XX}$, que realza las figuras parentales en cuanto a la conformación psíquica del niño (no ya en términos morales y sociales solamente), y a la luz de las cuales las posibilidades 
de los niños de desarrollarse y de ser escolarizados con éxito pasarán a depender de este nuevo reparto y atribución de responsabilidades adultas. Por otro lado, y unas décadas más tarde, esta ampliación de atribuciones y demandas es documentable en relación con el énfasis que adquirió - de la mano de la influencia de los organismos internacionales - la participación social y familiar, inéditas en momentos anteriores del siglo XX.

Por cierto, es importante también explicitar que, junto con estos procesos de cambio, encontramos algunas fuertes continuidades históricas cuya importancia no podemos soslayar. Por un lado, nos referimos a la significativa continuidad que hemos podido documentar en nuestra sociedad — evidenciada en los respectivos trabajos de camporespecto de la importancia y valoración social de la escolarización infantil por parte de amplios sectores de la población, que la ha ido apropiando como un derecho social (fuertemente reclamado y defendido). Por otro lado, junto con las múltiples transformaciones que se han producido, la educación (incluyendo la escolarización) y el cuidado de la infancia han sido indudablemente a lo largo del tiempo un campo de regulaciones, disputas y múltiples prácticas locales, con un fuerte involucramiento del Estado. Pero como planteamos, la complejidad de estos procesos de regulación de la vida familiar - a través de las definiciones sobre las responsabilidades adultas - incluye, pero rebasa ampliamente, la injerencia del Estado. ${ }^{12}$

En efecto, para ampliar nuestro desarrollo sobre los modos de regulación de las responsabilidades adultas en torno a los niños y nuestra discusión con el frente discursivo contemporáneo que ubicamos al principio, queremos a continuación, ahondar en los modos en que estas definiciones hegemónicas son tensionadas y apropiadas en escenarios locales donde se ponen en juego distintas acciones de cuidado infantil.

\section{El cuidado, la educación infantil y la diversidad de arreglos y prác- ticas en los escenarios locales}

En los diferentes contextos en que realizamos nuestro trabajo de campo, los grupos familiares con quienes nos contactamos desarrollan diversas prácticas destinadas a la crianza y la educación de los niños. Nos referimos a prácticas que tienen injerencia en esferas diversificadas de la vida de los hijos: el esparcimiento, la organización doméstica y también la escolaridad obligatoria. Entre nuestros entrevistados, en el marco de condiciones de vida claramente distintivas, encontramos continuidades que no podemos soslayar. Una de ellas, como dijimos, está relacionada con la importancia y valoración social que, de manera generalizada entre los distintos sectores sociales, se le otorga a la escolarización infantil -incluyendo a los sectores populares, sobre quienes recaen importantes estigmas acerca del interés por la escuela-.

Los hombres y mujeres con quienes conversamos, en reiteradas oportunidades, compartieron con nosotros su preocupación en torno a la educación de los hijos. Estos intercambios incluyen, de manera crucial y vívida, la alusión a la responsabilidad que les compete. La referencia a la serie de cumplimientos y deberes para con los niños se destaca por presentarse como un aspecto con importante sentido interiorizado, ${ }^{13}$ a la vez que insume cuotas significativas de trabajo cotidiano, el cual es llevado adelante desde diversos posicionamientos, resoluciones prácticas y condiciones de posibilidad. Entre los grupos familiares que conocimos, este sentido naturalizado acerca de lo que se "debe hacer" - pasible de vincular con los procesos anteriormente referidos sobre la producción social de la responsabilidad parental- abarca cierta exaltación de los progenitores por incluir de manera prioritaria las decisiones sobre la crianza y escolarización de los hijos. Como hemos documentado, un número importante - por no decir todos- de los padres que hemos entrevistado han sido rotundos al exponer cómo
12. Vale aclarar que de ninguna manera acá entendemos al Estado de un modo restringido a las instituciones y órganos de gobierno. Nos interesa más bien apuntar a una comprensión compleja del Estado, a través del análisis de sus efectos (en el sentido de Trouillot, 2001) y de sus modos de regulación moral (en el sentido de Corrigan y Sayer, 2007).
13. Aludimos a un sentido interiorizado que, como veremos, no va a contrapelo de la generación de apropiaciones activas por parte de estos mismos sujetos. 
una serie de acciones son de su exclusiva incumbencia (se destacan la selección de la escuela y las decisiones sobre la elección de las amistades y los circuitos de sociabilidad). Sin embargo, es una enunciación y premisa que se combina, en simultáneo, con otras muchas prácticas que alternan con esta misma exclusividad a la cual nos referimos.

Tal como adelantamos, en nuestro presente, la definición de responsabilidades adultas con respecto a los niños se encuentra fuertemente centrada alrededor de los padres y las madres. Es una centralidad que, como la etnografía lo ha documentado, no sólo resultaría muy extraña en otras sociedades que han desarrollado formas diferentes de atribución de responsabilidades en torno a las nuevas generaciones (Díaz de Rada, 2003; Cerletti, 2017), sino también para las resoluciones cotidianas que toman muchas familias, incluso aquellas organizadas bajo el modelo "nuclearizado". El trabajo de campo nos puso en contacto con un universo diversificado de conformaciones domésticas involucradas en la crianza y la educación de los chicos. Mientras que en algunas de estas conformaciones los niños estaban al cuidado de ambos progenitores, en otros casos, la crianza la llevaba adelante un sólo progenitor (generalmente la mujer), o bien - en un número alto - el/la progenitor/a con su nueva pareja. Pero, asimismo, nos encontramos con experiencias de crianza en las que, a pesar de estar llevadas adelante por ambos progenitores, como expusimos más arriba, diversos familiares coadyuvan simultáneamente en aspectos no siempre tangibles ni previstos. Además, podemos afirmar que, en cuanto a crianza y cuidado, uno de los aspectos a destacar es la heterogeneidad entre las decisiones y prácticas que los núcleos ponen en juego, independientemente de las composiciones a las que nos estamos refiriendo. A lo largo del trabajo de campo, hemos documentado decisiones muy variadas en cuanto a cómo los adultos se relacionan con la escuela y otras instituciones educativas de los niños, siendo incluso esta variación parte de la trayectoria a lo largo del tiempo de un mismo núcleo familiar y/o sujeto, hecho que hemos registrado puntualmente para el caso de familias con prole numerosa y generaciones distintas de hijos bien delimitadas. Esta es la experiencia de varias mujeres - y hombres-a los que conocimos, entre ellos, Teresa, una pobladora de 52 años que crio a sus hijos en uno de los barrios populares que conocimos. Ella nos decía:

T: Yo tuve 10 hijos en total. Yo tuve la camada de los chicos más grandes y la de los más chicos. Ahora estoy con los más chicos, el más grande de 15 y la nena más chica de 5. Pero todo es muy distinto a como crie a los primeros.

\section{E: ¿En qué fue distinto?}

T: Antes con los más grandes tuve que pelearla duro, todo fue más difícil, entre lo económico, pero que yo también tenía otra cabeza. No me importaba mucho la escuela, o si estaban bien comidos. Por ahí andaban solos. Ahora pienso distinto, estoy atrás para que no falten a la escuela, que la ropa esté bien. Eso me ayudaron a ver unas vecinas: que el estudio es importante, que es importante la escuela. Acá en el barrio se levantaron muchos lugares para ayudar a los chicos y nos ayudaron a nosotros a ver eso (Entrevista a Teresa, octubre de 2009).

Como sustenta lo documentado en las observaciones y las entrevistas, la construcción de sentido que producen las personas se nutre de valoraciones y perspectivas que difícilmente podamos escindir de la influencia recibida en los territorios y contextos donde se desarrollan sus vidas. Es importante decir que dichos escenarios (barrios, parajes, circuitos de circulación cotidianos), raramente están exentos de influencias externas y, por sobre todo, de las recuperaciones activas y selectivas que realizan los propios sujetos. 
En los hechos, las iniciativas vinculadas con la crianza y socialización de los hijos se desarrollan afrontando distintos desafíos, los cuales cobran especificidad acorde con las condiciones vinculadas con su clase social. En el caso de los sectores populares, la crianza y el cuidado de los hijos se produce paralelamente mientras sortean vicisitudes que incluyen la disputa por el espacio urbano, el acceso a la vivienda y la sobrevivencia diaria. En la realidad cotidiana de estos hombres y mujeres, la privación económica afecta negativamente el acceso a un conjunto de derechos que se entienden significativos en nuestras sociedades para el cuidado infantil. En el caso de las familias de clase media, las condiciones materiales para acceder al espacio urbano y la vivienda, así como para la sobrevivencia diaria (en sentido amplio), son claramente diferentes (aún con una gran diversidad de situaciones). En el acercamiento a unos y otros contextos, se hacen palpables las diferenciales marcas de la desigualdad social. A pesar de esto, muchos de nuestros entrevistados/as pertenecientes a la clase media no son de ningún modo ajenos a las constricciones materiales ni a las sobreexigencias que puede producir esta centralidad epocal en la responsabilidad de los padres y las madres por la crianza y la educación infantil, y los consecuentes procesos de individuación de las responsabilidades. Es significativo el modo en que Bibiana, una mujer de 34 años a quien conocimos en nuestro trabajo de campo con familias de clase media, explicaba las dificultades por las que había atravesado en relación con la crianza y la educación de su hija tras su divorcio. Luego de varios años y con una nueva pareja, había logrado mudarse a una casa espaciosa, en un barrio muy arbolado, mejorar sus condiciones laborales, y había podido cambiar a su hija a una escuela con la que estaba muy contenta. Respecto de todos estos cambios, afirmaba:

No es que antes no lo quería, antes no tenía las posibilidades (...). Ya el sólo hecho de tener que trabajar todo el día, dejarla con otra piba que te la cuide, que encima por ahí como económicamente no la podía dejar con quien quería, la dejaba con quien podía, que era mi vieja, que era gratis... (Entrevista a Bibiana, noviembre de 2016).

Muchas de las personas a las que entrevistamos relataron dificultades de este tipo, y también solían hacer alusión a lo "pesado" (en palabras de una de ellas) que podía resultar el desarrollo de la educación y cuidado de sus hijos, frecuentemente sin ayuda de otros adultos por fuera de la pareja.

Ahora bien, atender a la situación social de las familias no significa excluir del análisis la manera en que se posicionan los hombres y mujeres frente a estas vicisitudes, ni obviar sus estrategias tendientes al cuidado y la crianza de los hijos. Y pese a estar marcados por las condiciones de vida, los familiares de los niños accionan prácticas que relativizan cualquier intento por comprender las iniciativas sobre la crianza y la educación como hechos mecánicamente derivados de la posición social.

Como hemos registrado, cotidianamente, hombres y mujeres, en el marco de inserciones e interacciones diversas, ponen en juego estrategias y prácticas de diverso tipo. Mientras algunas prácticas - sin distinción de clase - se asocian a las peticiones contemporáneas (asistir a las reuniones, mirar los cuadernos, ayudar a completar la tarea), muchas otras aluden a formas de participación e involucramiento que, si sobresalen por algo, es por llevarse adelante a través de modalidades que se corren de los carriles y formatos institucionalizados por las escuelas (es decir, son prácticas que alteran los espacios y tiempos previstos por las instituciones, así como los encuadres comunicativos, entre otros).

Según pudimos reconstruir - y como ampliaremos en el apartado siguiente-, los adultos con niños a su cargo despliegan una diversidad de acciones en el marco de cambiantes configuraciones domésticas y en los territorios donde se vive, en todos los sectores sociales con los que trabajamos. Los procesos de educación y cuidado de los 
14. En la provincia de Buenos Aires, el contexto de la mayor parte de nuestro trabajo de campo, la elección de escuelas es libre, no se encuentra regulada por el Estado (Veleda, 2012). Según nuestros registros, los padres son los principales encargados de elegir la escuela a la que asistirán sus hijos. El proceso de selección de escuelas suele ser asumido por nuestros entrevistados como una responsabilidad indelegable, $y$ conjuga una serie muy compleja de condiciones de posibilidad y de preferencias, dinámicas y cambiantes a lo largo del tiempo. niños, así, se desarrollan dentro de estas diversas conformaciones, lo cual por cierto, como aludimos al principio, no redunda en resultados positivos o negativos a priori.

\section{Las definiciones acerca de las responsabilidades vistas desde la perspectiva de los adultos involucrados: confrontar sentidos}

Como venimos planteando, las madres y los padres (estos últimos, en menor medida) con quienes hemos entrado en contacto expresan de diversas formas y como un hecho indudable su protagonismo respecto de la educación de sus hijos e hijas. Sin embargo, los sentidos que les otorgan a sus responsabilidades $-\mathrm{y}$ a su repartición con otros actores- distan de ser homogéneos.

Para varios de nuestros entrevistados/as - pertenecientes, por cierto, a distintas clases sociales-, la educación de los niños se significa como un evento "compartido" entre la familia y las instituciones especializadas en la educación infantil, en el que la familia tiene que "acompañar" lo que sucede tanto en la escuela como en otros espacios educativos que, por su parte, esa familia eligió. ${ }^{14}$ Así, en sus prácticas discursivas aparece en primer plano la importancia de la familia en la educación, incluyendo la escolaridad. Con sentidos que evidencian una activa apropiación de - y retroalimentación con-aquellos hegemonizados en la contemporaneidad, estos adultos cotidianamente destacan la realización de un trabajo vinculado a las actividades escolares y al cuidado de los chicos y a lo realizado en las instituciones en cuestión, a través de acciones concretas, en buena medida condensadas en las categorías "sentarse" y "acompañar" (aludidas más arriba). De tal forma, esta responsabilidad compartida se despliega mediante dispositivos específicos (tales como el intercambio de anotaciones en los cuadernos escolares o el envío de ciertos materiales) y la rutina de observar las tareas escolares y completarlas en el espacio doméstico. Muchos de nuestros entrevistados/as responden de este modo a las frecuentes demandas de las instituciones educativas, y más aún, en algunos casos se hace claramente explícito el acuerdo con que esta es la modalidad en que debe conducirse "la familia" en relación con la escolaridad y las acciones de cuidado que otros desarrollan para con los hijos. Entre nuestros entrevistados, así lo narraba Paola, una madre con cuatro hijos en edad escolar, quien, de manera muy sacrificada, reparte su escaso tiempo entre las tareas de su hogar y el trabajo a tiempo completo como empleada doméstica en un barrio privado lindante a su domicilio:

Acá, muchos en el barrio miran para otro lado, como si la escuela tuviera que hacer las cosas sola. Yo me reparto en mil pedacitos. Me levanto temprano, a las 7 de la mañana ya tengo el almuerzo preparado y de ahí llevo a los chicos al colegio. Me meto a trabajar hasta que cae la noche. Cuando llego, lo primero que agarro son los cuadernos de los chicos. Ni bien veo una nota de alguna maestra, ya estoy pidiendo en el trabajo el día para poder ir. Cuando puedo, además, siempre doy una mano en la escuela. A veces no se puede y la escuela tiene que entender (Entrevista a Paola, abril de 2012).

Vale aclarar, sin embargo, que estos posicionamientos se sostienen de modos diversos, incluso en una misma familia. Tal es el caso de quienes eventualmente mencionan diferencias de apreciación con sus parejas respecto de la independencia o no de sus hijos para realizar sus actividades escolares, como Alma, una mujer de 39 años de clase media, quien expresaba algunas discrepancias con su marido, Francisco. Al hablar sobre el mayor de sus hijos, de ocho años en ese momento, explicaba que Francisco veía críticamente que el nene "no tiene armada la rutina, él no se sienta solo". Frente a esto, ella señalaba enfáticamente: 
'yno, gordo, no se sienta solo, qué sé yo, hay que sentarse [con el nene]'. Yo que fui siempre mucho más inquieta [en comparación con su marido], a mí me sentaron siempre hasta séptimo grado, al lado [de algún adulto], en el momento de hacer. A mí me parece que en eso tenemos que acompañar y considero yo que es parte de ambos, de la familia también, saber en qué andan, y qué hay que hacer, digamos (Entrevista a Alma, agosto de 2015).

En efecto, si bien por razones de espacio no podemos ampliarlo acá, es importante señalar que la producción de sentidos respecto de las obligaciones y responsabilidades adultas implica a su vez la definición de sentidos y posicionamientos sobre las responsabilidades de los niños (muchas veces también referidas a las diferentes edades de la vida).

Sin embargo, no todas las personas con las que trabajamos quedan incluidas en el planteo anterior. Para muchos de nuestros entrevistados/as, en efecto, la escolarización se entiende como un proceso en el que tienen más centralidad las propias responsabilidades de los niños y los/as docentes. De tal forma, y a diferencia de los casos anteriores, varios de nuestros entrevistados/as generan un posicionamiento firme $-\mathrm{y}$ eventualmente explicitado frente a los docentes, coordinadores y directivos- respecto de no involucrarse en las actividades escolares de los niños. Cecilia y Matías, una pareja de unos cuarenta años a la que entrevistamos en el marco de nuestro trabajo de campo con familias de clase media, lo expresaban así:

C: ¡Ah! y en este colegio [al que mandan a sus dos hijos actualmente] le preguntamos a Norma [la directora], le dijimos: '¿vamos a tener que hacer actividades? Porque nosotros NO queremos'. Dijo 'no, quédense tranquilos que el que quiere, quiere, y el que no quiere, no'. Y nosotros, NO.

E: ¿Y no los convocan a actividades extra?

C: Te convocan si querés.

M: Claro [se superponen], convengamos que nosotros...

C: Te convocan si querés, te anotás si querés. Ahora, para el Día del Maestro vino una notita, 'el que quiere participar...', yo pongo NO (Entrevista a Cecilia y Matías, agosto de 2015).

Los sentidos que transmiten estos sujetos en torno a las responsabilidades adultas e infantiles implican la definición de límites bastante precisos, dentro de las cuales una serie de acciones (supervisar las carpetas y las tareas) es competencia de los niños, mientras que otras (corregírselas, enseñarles, etc.) es responsabilidad de los docentes y educadores, y en todo caso, los padres ayudan con las actividades escolares y las que demandan las instituciones al principio de la escolaridad y el ciclo de inicio, y luego ya no. Sin embargo, es importante no asumir explicaciones lineales o simplistas respecto de esta caracterización de los diversos posicionamientos. Así, si bien en estos casos podría parecer que implica menos cantidad de trabajo de parte de los adultos a cargo de los niños, especialmente en familias pertenecientes a la clase media, hemos documentado situaciones en las que este modo de diferenciar las responsabilidades va de la mano de un sentido respecto de la educación de acuerdo con el cual la escolaridad se busca como un aspecto más acotado en la vida de los niños (por caso, mediante la elección de una escuela de jornada simple). Y luego, por fuera de la escuela, muchos niños asisten a una gran cantidad de actividades recreativas, deportivas, de enseñanza de otros idiomas, a centros de apoyo escolar, etc. 
15. Esta pedagogía se basa en los postulados del filósofo austríaco Rudolf Steiner (fundador de la filosofía Antroposófica). La presencia de "escuelas Waldorf" en la Argentina data de finales de la década de 1960, y han crecido en número especialmente en las últimas décadas, con mayor concentración en la zona norte del conurbano bonaerense. Se particularizan por un énfasis en la relación de los niños con la naturaleza y con la espiritualidad, y con especial dedicación a la expresión artística. Los niños cursan todos los grados con un/a mismo/a docente, y en el nivel inicial se integran niños de distintas edades. Estas son, entre otras, algunas de sus características, que, por razones de espacio, no podemos desarrollar acá.
Si bien, como dijimos, algunos de estos adultos se involucran relativamente poco en la escolaridad de sus hijos - en términos de actividades adultas vinculadas a las tareas y actividades escolares de los niños- y buscan que la escuela tampoco les insuma demasiado tiempo, sin embargo, sostienen cuotas muy importantes de trabajo cotidiano (especialmente las mujeres), llevándolos y trayéndolos a todo el amplio abanico de actividades extraescolares que realizan (usualmente elegidas por los niños).

En otros casos, hemos documentado sentidos en torno a la educación como una cuestión centralmente vinculada a los padres y las madres - a "las familias" - ; sentidos que surgen a su vez en relación con prácticas tales como construir establecimientos escolares, o de elegir aquellos (usualmente de pedagogía "Waldorf", ${ }^{15}$ en el caso de las familias pertenecientes a la clase media) donde se valora muy positivamente el lugar de las familias en el sostenimiento cotidiano de las escuelas, eventualmente desde su fundación (incluso más allá de la regulación estatal). Las modalidades de involucramiento que estos adultos despliegan respecto de la educación escolar de sus hijos, que se produce cotidianamente asumiendo distintas responsabilidades, implica márgenes muy difusos entre familia y escuela: las familias aportan, además de dinero, una importante cantidad de trabajo vinculado al mantenimiento y sostenimiento de la escuela, y tienen una presencia cotidiana que se cristaliza en la realización de múltiples actividades que las involucran. Y en algunos casos, además, la presencia cotidiana termina derivando en que formen parte de la escuela como docentes. En esta línea, nos resultó particularmente significativa una experiencia que decidieron llevar adelante un grupo de padres (se conocieron en un "espacio de juegos" "Waldorf” para niños de tres a cinco años), que optaron por organizar un espacio propio concerniente a la misma pedagogía para la escolaridad primaria de sus hijos. Uno de los padres cedió lugar en su casa, el resto trabajó y coordinadamente armaron un aula, contrataron a una maestra "Waldorf”, y ese grupo de niños continuó ahí su escolaridad. Este espacio está compuesto por chicos de distintas edades y se sostiene con el trabajo de ese grupo específico de padres. Por otro lado, nunca tuvo ningún tipo de reconocimiento estatal, motivo por el cual son sumamente cuidadosos con la difusión de su experiencia (fue determinante la importancia del anonimato de nuestro trabajo de campo). La decisión que tomó este grupo de padres implica un aporte económico y en tiempo de trabajo muy significativo, y que, dadas las características de la experiencia, no pueden dejar de hacer. Se trata de una práctica escolar infantil que pone el foco del sostenimiento en la responsabilidad de los padres, vinculada con un posicionamiento que conlleva una cuota de ilegalidad, por lo complejo de regular la situación con el Estado.

Ahora bien, para cerrar el análisis, el transcurso de la indagación nos puso frente a otras construcciones de la atribución de responsabilidades que alteran el planteo naturalizado y más difundido de que la distribución de deberes y obligaciones se dirime de manera exclusiva dentro del binomio familia/ escuela. En los escenarios donde investigamos -especialmente en los barrios populares-, padres y adultos familiares de los niños protagonizan - como gestores o partícipes activos- de la creación y el desarrollo de espacios escolares y educativos levantados en las inmediaciones del lugar en que viven, llevados adelante por los propios vecinos. Progresivamente, en contextos ubicados en la periferia urbana, grupos de pobladores - con una mayoría de mujeres - fueron asumiendo un lugar activo en la resolución de varios de los requerimientos a los que aludimos, relativos al acompañamiento y cumplimiento de responsabilidades vinculadas a la escolaridad y el cuidado de los chicos. Basándose en las experiencias creadas por militantes y voluntarios externos a los barrios en el retorno de la democracia a fines de 1983, se abrieron espacios colectivos tales como merenderos, apoyos escolares, centros para la recreación y también guardería para los niños más pequeños, estructurados en formatos que exceden ampliamente a las usuales colaboraciones entre "familiares" e incluso la ayuda mutua entre vecinos. Varios de los hombres y mujeres, padres y tutores de los chicos con quienes conversamos fueron contundentes al momento de situar a 
estas experiencias como muy significativas para resolver los requerimientos escolares relacionados con sus hijos, ya sea delegando a otros vecinos algunas obligaciones propias, o siendo partícipes activos de las iniciativas. Rosa, una mujer de mediana edad, a quien entrevistamos mientras colaboraba en el merendero del barrio-el mismo donde sus hijos recibían una copa de leche-, no escatimó palabras al momento de hablar del centro de apoyo escolar levantado por sus vecinos:

Para mí el apoyo es una gran ayuda, porque es un beneficio muy importante para mis hijos también. Yo llegué hasta quinto grado, tenía 14 años y abandoné el colegio. Yo no puedo seguir muchas cosas de la escuela de mis hijos. Entonces, acá, Gloria y Roberto, y las demás vecinas le dan una ayuda a los chicos en las tareas que piden las maestras. Es muy bueno porque también hacen otras cosas, consiguen paseos que yo no puedo hacerles, por ejemplo y muchas más cosas: tienen arte, cocina y deporte. Además, muchas veces nos juntamos los papás y hacemos algo también. O vienen de otro lado a darnos una charla sobre cómo criar a los chicos y cosas así. Es una ayuda importante para sacar los chicos adelante, sobre todo con las penurias que pasamos acá en el barrio en lo económico, ¿no? (Entrevista a Rosa, noviembre de 2006).

En paralelo, incluso en relación con las iniciativas que les "competen" a las figuras parentales, documentamos una serie de acciones directas que los propios referentes barriales llevan adelante junto con la escuela, y también en relación con los padres de los niños. Nos referimos a eventos puntuales en los que los padres delegan o bien son secundados por pobladores a cargo de los espacios de merendero y apoyo escolar para plantear determinadas cuestiones a los maestros y directivos de la escuela. Por cierto, en estas audiencias improvisadas sobresale la capacidad de varios referentes barriales para neutralizar algunas decisiones de la escuela, y en otros casos, además, estos mismos referentes logran disuadir ciertas posturas renuentes de los padres, sobre todo de aquellos que mantienen una relación más marginal respecto de los centros. Según entendemos, estas iniciativas en las cuales las responsabilidades se colectivizan abren sentidos más diversos a las visiones hegemónicas sobre la repartición de los deberes y las obligaciones adultas.

\section{Palabras finales}

Con la apelación a la participación, el interés y el acompañamiento, los discursos que han hegemonizado el campo de la educación y el cuidado infantil han perfilado un modo de regulación de la vida familiar que supone un novedoso reparto de las responsabilidades adultas. Esto, a su vez, provocó algunas transformaciones significativas. Este relativamente nuevo reparto de responsabilidades da por sobreentendidas varias cuestiones que no podemos soslayar. Entre otras, presupone la disponibilidad adulta para este tipo de prácticas, centraliza la responsabilidad adulta respecto de los niños en torno a las figuras parentales y reordena una modalidad de interacción entre padres, educadores y otros cuidadores de los niños. Lejos de ser naturales, se trata de postulados cuya historicidad es posible documentar, y, por lo tanto, desnaturalizar y problematizar.

Efectivamente, este frente discursivo contemporáneo ha tenido sin duda una significativa productividad, en tanto hemos evidenciado los modos en que es cotidianamente apropiado y reproducido por un amplio conjunto de actores. Sin embargo, esas apropiaciones son por cierto activas, y por tanto, transformativas, de modo que se van instalando a su vez, en los escenarios locales de actuación, sugestivos matices y diferencias. Asimismo, hemos documentado que junto con - y más allá de - la extensión que estos postulados hegemónicos han alcanzado en el campo de la educación y el cuidado infantil, los adultos cotidianamente vinculados a los niños — sus padres, madres, docentes, 
educadores, parientes, vecinos, etc.- producen también sentidos diversificados respecto de la atribución de responsabilidades correspondientes a sus progenitores, así como a otros sujetos (individuales y colectivos). De tal forma, por contrapartida a la fijeza y naturalidad con que desde este frente discursivo se instalan modos sumamente individualizados de atender a la formación y reproducción de las nuevas generaciones, en las prácticas cotidianas que hemos registrado, los sujetos producen otros modos de significar las obligaciones y responsabilidades frente a los niños, y por cierto, también por parte de los mismos niños. En esos otros modos permean diversas aristas de estos lineamientos hegemónicos, al tiempo que son confrontados y resignificados tanto en iniciativas colectivas que asumen de formas más compartidas estas responsabilidades, como en las maneras de hacer cotidianas que los adultos encuentran para vincularse con la educación (incluyendo la escolarización) y la crianza de sus hijos.

Asimismo, como hemos visto, estos discursos hegemónicos presuponen una importante disponibilidad de las "familias", lo cual se concreta en el énfasis sobre las responsabilidades parentales, pero sin poner en consideración las condiciones de posibilidad -ni tampoco las preferencias- con las que cuentan los sujetos concretos. En este sentido, todas las personas con las que trabajamos han tenido situaciones cambiantes para afrontar estas responsabilidades. Las condiciones laborales, habitacionales, organizacionales y la disponibilidad de tramas relacionales que contribuyen al despliegue del trabajo reproductivo vinculado a la educación infantil se imbrican plenamente entre sí, de manera que la transformación en alguna de estas dimensiones posibilita o moviliza modificaciones en las otras, de un modo indisociable a su vez de las prácticas cotidianas vinculadas a la educación y la crianza de los niños. Estas últimas, así, se van desplegando al calor de estas imbricaciones, por lo que es imposible establecer regularidades en la primacía de una de estas dimensiones por sobre las otras. Es decir, lo que reflejan los avances de nuestros trabajos con sujetos pertenecientes a distintas clases sociales es que las prioridades son dinámicas - incluyendo las preferencias educativas-, y se van adecuando en relación con la compleja articulación de diversas dimensiones vitales. Como planteamos, en los cambiantes contextos donde tienen lugar estas articulaciones, los adultos activamente producen - se apropian, tensionan, contraponen - sentidos respecto de las responsabilidades y obligaciones - propias y ajenas - en torno a la educación y el cuidado de sus hijos, de modos que no permiten prever causalidades directas entre esos sentidos y las prácticas con las que se despliegan, y los resultados en las experiencias formativas infantiles.

\section{Notas}

1 En nuestros planteos, el término crianza, a diferencia de la tendencia presente en estudios sociológicos ligados a determinadas corrientes de la psicología y a la biomedicina, no se restringe ni al momento de la lactancia de los niños ni se acota a un período etario (marcado como los primeros años de vida), sino que comprende un conjunto amplio de prácticas y relaciones que los adultos identifican para con la atención infantil (y que puede comprometer acciones relativas a la formación, el cuidado, el esparcimiento, la salud, la alimentación). Asimismo, nuestro interés por la crianza y las experiencias formativas se enlaza de manera inescindible con las construcciones sociales sobre el cuidado. Dentro de nuestros planteos, la acción de cuidar al otro no excluye educar, y en el acto de educar, es posible prever ciertas formas diversificadas del cuidado (Cerletti, 2014; Santillán, 2017). 


\section{Q Referencias bibliográficas}

》 ACHILLI, Elena. 2005. Investigar en Antropología Social. Los desafíos de transmitir un oficio. Rosario: Laborde Editor.

" ACHILLI, Elena. 2010. Escuela, familia y desigualdad social. Rosario: Laborde Editor.

" AMUCHÁSTEGUI, Marta. 2000. “El orden escolar y sus rituales”. En: S. Gvirtz (Comp.). Textos para repensar el día a día escolar. Buenos Aires: Santillana. pp. 59-77.

» BATALLÁN, Graciela. 2007. Docentes de infancia. Antropología del trabajo en la escuela primaria. Buenos Aires: Paidós.

" CARLI, Sandra. 1997. "Infancia, psicoanálisis y crisis de generaciones. Una exploración de las nuevas formas del debate en educación”. En: A. Puiggrós (Dir.). Dictadura y utopías en la historia reciente de la educación argentina (1955-1983). Buenos Aires: Galerna. pp. 221-287.

》 CARLI, Sandra. 2005. Niñez, pedagogía y política. Transformaciones de los discursos acerca de la infancia en la historia de la educación argentina entre 1880 y 1955. Buenos Aires: Miño y Dávila.

" CARMONA, Carlos Alonso. 2014. "Familia escuela y clase social: sobre los efectos perversos de la implicación familiar”. RASE, 7(2): 395-409.

»CERLETTI, Laura. 2012. “'Familias’ y 'participación’: un análisis comparativo de la Ley 1.420, la Ley Federal de Educación y la Ley de Educación Nacional”. Revista Propuesta Educativa, 37: 69-77.

》CERLETTI, Laura. 2014. Familias y escuelas. Tramas de una relación compleja. Buenos Aires: Biblos.

" CERLETTI, Laura. 2015. "Del presente a los años 60: representaciones y regulaciones sobre la vida familiar y la educación infantil”. Revista de Antropología Social, 24: 349-374.

»CERLETTI, Laura. 2017. “'De ellos soy padre y madre'. Responsabilidades sobre la educación infantil en situaciones de monoparentalidad”. Revista Pilquén - Sección Psicopedagogía, 14(2): 30-39.

" CERLETTI, Laura y GESSAGHI, Victoria. 2017. “¿Niños pobres? ¿Niños ricos? Discusiones sobre las representaciones y regulaciones de la vida familiar en torno a la escolaridad infantil”. En: G. Novaro, L. Santillán, A. Padawer y L. Cerletti (Coords.). Niñez, regulación estatal y procesos de identificación. Buenos Aires: Biblos. pp. 45-72.

» CERLETTI, Laura y SANTILLÁN, Laura. 2013. "Educación y escolarización infantil: aportes etnográficos para analizar la configuración de orientaciones hegemónicas y las iniciativas familiares y comunitarias (Área Metropolitana de Buenos Aires)". XIII Simposio Interamericano de Etnografía de la Educación. Los Ángeles: UCLA.

" CORRIGAN, Philip y SAYER, Derek. 2007. "El Gran Arco: La formación del Estado inglés como revolución cultural”. En: M. Lagos y P. Calla (Eds.). Antropología del Estado. Dominación y prácticas contestatarias en América Latina. Bolivia, La Paz: INDH/PNUD. pp. 36-116.

" COSSE, Isabella. 2010. "Argentine Mothers and Fathers and the New Psychological Paradigm of Child- Rearing (1958-1973)”. Journal of Family History, 35(2): 180-202.

" CRAGNOLINO, Elisa. 2000. "La dimensión histórica en una investigación de antropología educativa”. Cuadernos de Antropología Social, 12: 73-95. 
" DARRÉ, Silvana. 2013. Maternidad y tecnologías de género. Buenos Aires: Katz.

» DíAZ DE RADA, Ángel. 2003. “Las edades del delito". Revista de Antropología Social, 12: 261-286.

"DI NELLA, Dino. 2016. “Familias monoparentales y responsabilidad parental. Un análisis sociojurídico". Arxius de Ciènces Socials, 34: 11-28.

»EDWARDS, Rosalind y ALLDRED, Pam. 200o. "A typology of parental involvement in education centring on children and young people: negotiating familialisation, institutionalization and individualization". British Journal of Sociology of Education, 21(3): 435-455.

"FONSECA, Claudia y CARDARELLO, Andrea. 2006. "Direitos dos mais e menos humanos”. En: C. Fonseca y P. Schuch (Comps.). Políticas de proteção à infancia. Um olhar antropológico. Porto Alegre: Editora UFRGS. pp. 219-251.

» HARRIS, Leslie. 2009. "Making parents pay: Understanding parental responsibility laws". Family Advocate, 31(3): 38-42.

» MENÉNDEZ, Eduardo y SPINELLI, Héctor. 2006. Participación social. ¿Para qué? Buenos Aires: Lugar editorial.

» MORENO, José Luis. 2002. La política social antes de la política social. Buenos Aires: Prometeo.

» NARI, Marcela. 2004. Políticas de maternidad y maternalismo político. Buenos Aires, 18901940. Buenos Aires: Biblos.

" NEUFELD, María Rosa. 1997. “Acerca de antropología social e historia: una mirada desde la antropología de la educación". Cuadernos del Instituto Nacional de Antropología y Pensamiento Latinoamericano, 17: 145-158.

"NEUFELD, María Rosa. 2000. "Familias y escuelas: la perspectiva de la antropología social". Ensayos y Experiencias, 36: 3-13.

"NEUFELD, María Rosa y THISTED, Ariel. 2004. "Vino viejo en odres nuevos: acerca de educabilidad y resiliencia”. Cuadernos de Antropología Social, 19: 83-100.

» NOGUEIRA, Maria Alice. 2011. “A categoria 'família' na pesquisa em sociologia da educação: notas preliminares sobre um processo de desenvolvimento". Revista Inter-legere, 9: 156-166.

» OCHS, Elinor e IZQUIERDO, Carolina. 2009. "Responsibility in Childhood: Three developmental trajectories". Ethos, 37(4): 391-413.

» OCHS, Elinor y KREMER-SADLIK, Tamar. 2013. Fast-forward family. Home, work and relationships in middle-class America. Berkeley: University of Carlifornia Press.

»PLOTKIN, Mariano. 2003. Freud en las pampas. Orígenes y desarrollo de una cultura psicoanalítica en la Argentina (1910-1983). Buenos Aires: Sudamericana.

» ROCKWELL, Elsie. 2009. La experiencia etnográfica. Historia y cultura en procesos educativos. Buenos Aires: Paidós.

" RUSTOYBURU, Cecilia. 2010. "Entre reflexivas y madres patógenas. Las mujeres en los discursos de la Escuela para Padres en los años 1960". En: N. Álvarez (Comp.). Familias, género y después... Itinerarios entre lo público, lo privado y lo íntimo. Rosario: Prohistoria. pp. 53-78.

" SANJEK, Roger. 1991. “The ethnographic present”. Man, 26: 609-628.

"SANTILLÁN, Laura. 2009. "De responsabilidades y demandas que cambian”. Revista Novedades Educativas, 222: 14-17. 
» SANTILLÁN, Laura. 2012. Quiénes educan a los chicos. Trayectorias educativas, infancia y desigualdad. Buenos Aires: Biblos.

» SANTILLÁN, Laura. 2016. “Las ideas sobre el 'buen comienzo' y la primera infancia: construcciones hegemónicas y apropiaciones locales”. Revista Archivos de Ciencias de la Educación, 12(2): 217-235.

»SANTILLÁN, Laura. 2017. “¿Quiénes educan a los chicos? Una mirada desde la antropología sobre el cuidado, la enseñanza y la educación”. En: P. Redondo y E. Antelo (Eds). Encrucijadas de la educación infantil: entre el cuidar y el enseñar. Rosario: Homo Sapiens. pp. 17-35.

» TROUILLOT, Michel. 2001. “La Antropología del Estado en la era de la globalización”. Current Anthropology, 42(1): 225-231.

»VELEDA, Cecilia. 2012. La segregación educativa. Entre la fragmentación de las clases medias y la regulación atomizada. Buenos Aires: La Crujía.

\section{Otras fuentes}

» ORGANIZACIÓN INTERNACIONAL DEL TRABAJO (OIT). 2012. Un buen comienzo. La educación y los educadores en Primera Infancia. Informe para el debate en el Foro de diálogo mundial sobre las condiciones del personal de la educación de la primera infancia. Ginebra: Oficina Internacional del Trabajo, OIT.

»UNESCO. 2004. Participación de las familias en la educación infantil latinoamericana. Chile: Organización de las Naciones Unidas para la Educación, la Ciencia y la Cultura.

»UNESCO. 2012. Interacción escuela-familia. Insumos para las prácticas escolares. Brasil: Organización de las Naciones Unidas para la Educación, la Ciencia y la Cultura.

»UNICEF. 2002. Las escuelas y las familias por la educación. Guía de Orientación para el Trabajo en Talleres. Argentina: Fondo de las Naciones Unidas para la Infancia.

»UNICEF. 2005. Hacia una política pública en desarrollo infantil temprano. Las buenas prácticas. Argentina: Fondo de las Naciones Unidas para la Infancia.

¿UNICEF. 2009. Acercando las familias a la escuela. Chile: Fondo de las Naciones Unidas para la Infancia. 
